\title{
Transcranial Direct Current Stimulation (tDCS) of Left Parietal Cortex Facilitates Gesture Processing in Healthy Subjects
}

\author{
Peter H. Weiss, ${ }^{1,2 *}$ Elisabeth I. S. Achilles, ${ }^{1,2 *}$ Katharina Moos, ${ }^{1,2}$ Maike D. Hesse, ${ }^{1,2}$ Roland Sparing, ${ }^{1,2}$ and \\ Gereon R. Fink ${ }^{1,2}$ \\ ${ }^{1}$ Cognitive Neuroscience, Institute of Neuroscience and Medicine (INM-3), Research Center Jülich, 52425 Jülich, Germany, and ${ }^{2}$ Department of Neurology, \\ Cologne University Hospital, Cologne, 50924 Köln, Germany
}

\begin{abstract}
Gesture processing deficits constitute a key symptom of apraxia, a disorder of motor cognition frequently observed after left-hemispheric stroke. The clinical relevance of apraxia stands in stark contrast to the paucity of therapeutic options available. Transcranial direct current stimulation (tDCS) is a promising tool for modulating disturbed network function after stroke. Here, we investigate the effect of parietal tDCS on gesture processing in healthy human subjects. Neuropsychological and imaging studies suggest that the imitation and matching of hand gestures involve the left inferior parietal lobe (IPL). Using neuronavigation based on cytoarchitectonically defined anatomical probability maps, tDCS was applied over left IPL-areas PF, PFm, or PG in healthy participants $(n=26)$. Before and after tDCS, subjects performed a gesture matching task and a person discrimination task for control. Changes in error rates and reaction times were analyzed for the effects of anodal and cathodal tDCS (compared with sham tDCS). Matching of hand gestures was specifically facilitated by anodal tDCS applied over the cytoarchitectonically defined IPL-area PFm, whereas tDCS over IPL-areas PF and PG did not elucidate significant effects. Taking into account tDCS electrode size and the central position of area PFm within IPL, it can be assumed that the observed effect is rather the result of a combined stimulation of the supramarginal and angular gyrus than an isolated PFm stimulation. Our data confirm the pivotal role of the left IPL in gesture processing. Furthermore, anatomically guided tDCS of the left IPL may constitute a promising approach to neurorehabilitation of apraxic patients with gesture processing deficits.
\end{abstract}

\section{Introduction}

Many left-hemispheric stroke patients suffer from apraxia (Donkervoort et al., 2000), a disorder of motor cognition, which cannot solely be explained by elementary deficits of the sensorimotor system, verbal communication deficits, or lack of motivation (Goldenberg, 2008; Frey et al., 2011). Apraxia impairs activities of daily life and interferes with neurorehabilitation (Hanna-Pladdy et al., 2003; Pomeroy et al., 2011). Among other symptoms, apraxic patients commonly exhibit deficits in gesture processing. They are, for example, impaired in imitating and matching of meaningless hand gestures (Goldenberg, 1999). In healthy subjects, hand gesture matching leads to increased activ-

Received Oct. 5, 2012; revised Sept. 26, 2013; accepted 0ct. 2, 2013.

Author contributions: P.H.W., E.I.S.A., R.S., and G.R.F. designed research; P.H.W., E.I.S.A., and K.M. performed research; P.H.W., E.I.S.A., M.D.H., and R.S. analyzed data; P.H.W., E.I.S.A., K.M., M.D.H., R.S., and G.R.F. wrote the paper.

We thank our subjects, the four models (Barbara Achilles, Janina Deying, Veronika Betz, Maximiliane Betz), and K. P. Schmidt (Medizin Foto Köln, MFK) for their support; and our colleagues at the Institute of Neuroscience and Medicine, especially Dr. Anna Dovern and Dr. Ralph Weidner (for his valuable statistical counseling).

The authors declare no competing financial interests.

*P.H.W. and E.I.S.A. contributed equally to this work.

Correspondence should be addressed to Dr. Peter H. Weiss, Cognitive Neuroscience, Research Centre Jülich (INM-3), Leo-Brandt-Strasse 5, 52425 Jülich, Germany. E-mail: P.H.Weiss@fz-juelich.de.

DOI:10.1523/JNEUROSCI.4714-12.2013

Copyright $\odot 2013$ the authors $\quad 0270-6474 / 13 / 3319205-07 \$ 15.00 / 0$ ity in the left inferior parietal lobe (IPL) (Hermsdörfer et al., 2001), a common lesion site in apraxia (Dovern et al., 2011).

To date, therapeutic options for apraxia remain scarce despite its significant impact upon neurorehabilitation (Pomeroy et al., 2011; Vanbellingen and Bohlhalter, 2011; Cantagallo et al., 2012; Dovern et al., 2012). Transcranial direct current stimulation (tDCS) is a promising tool for modulating disturbed network function and the amelioration of cognitive deficits after stroke (for neglect, see Sparing et al., 2009). In tDCS, weak currents are applied to the cortex via two electrodes placed on the scalp. Anodal tDCS increases cortical excitability of the brain region under the stimulating electrode, whereas cathodal tDCS decreases cortical excitability (Nitsche and Paulus, 2000; Stagg and Nitsche, 2011). These cortical excitability changes may outlast stimulation up to a few hours (Sparing and Mottaghy, 2008). Recent studies suggest that neuronavigation may be helpful to fully exploit the potential of tDCS in modulating higher cognitive functions in humans (Moos et al., 2012).

The current study aimed at modulating gesture processing in healthy subjects using tDCS and adopting neuronavigation based upon cytoarchitectonically defined anatomical probability maps. We hypothesized that anodal tDCS applied over the left IPL improves gesture processing, thereby opening new avenues for the neurorehabilitation of gesture processing deficits in apraxia.

As neuropsychological and neuroimaging studies implicate different subareas of the left IPL in human gesture processing, 
either the left supramarginal gyrus (Hermsdörfer et al., 2001; Decety et al., 2002; Mühlau et al., 2005) or the left angular gyrus (Goldenberg and Hagmann, 1997; Rumiati et al., 2005), we applied frameless stereotactic neuronavigation in healthy participants $(n=26)$ to center tDCS over the three cytoarchitectonically defined areas PF, PFm, or PG of the left IPL. Thus, tDCS was focused on the left supramarginal gyrus (tDCS over PF), on the left angular gyrus (tDCS over PG), or on both gyri (tDCS over PFm) to assess possible neuroanatomically specific tDCS effects.

Specifically, before and after tDCS, subjects performed an experimental gesture matching task and, for control, a person discrimination task. Improvement of task performance due to (anodal, cathodal, or sham) tDCS dependent upon the specific stimulation site was assessed by changes in error rates and reaction times (RTs; before vs after tDCS).

\section{Materials and Methods}

Participants. Thirty healthy right-handed subjects (age, mean $\pm \mathrm{SD}, 25.6 \pm 4.4$ years, 14 women) participated in the study. Subjects received monetary allowance for their participation. The study was performed in accordance with standard safety guidelines (Nitsche et al., 2003) and the declaration of Helsinki. The study was approved by the local ethics committee and all participants gave written informed consent.

Experimental stimuli and tasks. Based on previous studies (Goldenberg, 1999; Hermsdörfer et al., 2001), we adopted a task for assessing the matching of hand gestures (gesture matching task). For control, subjects were asked to decide whether the same or a different woman performed those gestures (person discrimination task) (Hermsdörfer et al., 2001).

To create the stimuli, photos of four young female subjects each performing 10 meaningless hand gestures (Goldenberg, 1995) were taken from three different perspectives (front, semiprofile right and left). This constituted a set of 120 photos (digitally processed with the software Adobe Photoshop), based upon which 320 stimuli were created, each consisting of photo pairs showing either the same gesture twice $(50 \%)$ or two different gestures (50\%; for example stimuli, see Fig. 1A). Of these 320 stimuli, 288 stimuli were selected based on RTs and error rates of 25 healthy subjects (not enrolled in the tDCS study) performing the gesture matching task in a pilot experiment. These 288 stimuli were complemented by additional 288 stimuli, which were created by switching the presentation side of the photos within the stimuli (i.e., the two photos switched sides). The resulting total of 576 stimuli was divided into 6 subsets of 96 stimuli each for the gesture matching task. (Within each subset, half of the stimuli showed the same gesture twice and the other half two different gestures.) Thereafter, the same 576 stimuli were again divided into 6 subtests of 96 stimuli for the person discrimination task so that in each subset the stimuli showed the same person twice $(50 \%)$ or two different persons $(50 \%)$. Thus, both tasks were performed on the identical stimulus material (i.e., 576 stimuli each). Moreover, we ensured that within a given set only stimuli were included that triggered a differential response depending on the task at hand. Presentation of stimuli within sets and the order of the 6 stimulus sets were pseudo-randomized (Fig. 1B).

Participants were comfortably seated in a dimly lit room in front of a computer screen (14.1" TFT screen, viewing distance $70 \mathrm{~cm}$ ). Using Presentation Version 14.2 (Neurobehavioural Systems), one of the 6 stimulus sets (containing 192 photo pairs/stimuli) was presented to the subject. Within each set, half of the stimuli (i.e., 96 stimuli) were used for the gesture matching task and the other half for the control task (person discrimination). Depending on the task at hand, subjects pressed (as quickly and as accurately as possible) a button with their left index finger if the gestures (or persons) on the screen were the same and another button with their left middle finger if the gestures (or persons) were different. The 96 stimuli used for each task were divided into 4 blocks of 24 stimuli/photo pairs each in such a way that there was the same number of index and middle finger responses within each block of trials. For a given block of trials (24 stimuli), subjects performed either the gesture matching or the person discrimination task. Before the start of a new block, instructions that indicated the forthcoming type of task were pre- 
sented until the subjects pressed a button to continue. To ensure task adherence, during blocks subjects were reminded about the task to be performed (gesture matching, person discrimination) by a small colored letter cue ( $\mathrm{G}, \mathrm{P})$ displayed between the photos (Fig. 1A, blocks of trials). The two tasks were presented in alternating order (i.e., consisting of 24 stimuli) to ensure that any putative decline of tDCS effects over time was equally present for both tasks. Based on the subjects' left hand responses, RTs and error rates were assessed. After the subjects' response, the next stimulus was presented (with randomized interstimulus intervals of 800 , 900 , or $1000 \mathrm{~ms}$ ). Participants were asked to use their left hand to avoid any putative interference due to an unspecific modulation of left hemisphere motor areas by tDCS.

Directly after tDCS (see below), subjects performed a second set (of the 6 sets, each containing 192 stimuli), including both tasks. During the course of the experiment, each of the 6 sets was presented only once to each participant.

Experimental design. The within-subject factors STIMULATION (three levels: anodal tDCS, cathodal tDCS, and sham tDCS) and TASK (two levels: gesture matching task and person discrimination task) as well as the between-subject factor stimulation SITE [three levels: IPL-areas PF $(n=8)$, PFm $(n=8)$, and PG $(n=10)]$ constituted the experimental design. The study consisted of three sessions performed on 3 different days (Fig. 1B). The interval between sessions was at least $48 \mathrm{~h}$ (mean interval $80.8 \mathrm{~h}$ [SD $42.5 \mathrm{~h}$ ) (Moos et al., 2012), to avoid any potential tDCS carryover effects (Nitsche et al., 2008). Sessions were scheduled at similar daytimes for each subject.

Each session started with a practice block to familiarize the subjects with the setup and tasks (64 stimuli not used in the 6 experimental stimulus sets). Thereafter, subjects performed the task with one of the 6 stimulus sets. Then, anodal, cathodal, or sham tDCS was applied for 20 min (see below). Directly after tDCS, subjects performed the two tasks again with a second (i.e., different) stimulus set. Changes in error rates and RTs before tDCS and after tDCS served as the outcome measures for further analysis. The mean duration of the stimulus sets was $13.5 \mathrm{~min}$.

$t D C S$. tDCS procedures were adopted following Moos et al. (2012). In particular, neuronavigated tDCS (see below) was applied by a batterydriven constant DC current stimulator (neuroConn). A $25 \mathrm{~cm}^{2}$ stimulation electrode (to which polarity refers) was placed at the target area of stimulation using electrode crème (GE Healthcare) as a conducting medium and rubber bandages to hold the electrodes in place. The $35 \mathrm{~cm}^{2}$ reference-electrode was placed over $\mathrm{Cz}$ as in Sparing et al. (2009).

tDCS was applied in accordance with current safety guidelines (Nitsche et al., 2003). The type of tDCS [i.e., anodal (a), cathodal (c), or sham (s) tDCS] was pseudo-randomized across sessions. The pseudorandomization ensured that the six possible different stimulation orders (cas, csa, sca, sac, acs, and asc) occurred similarly often. A constant current of $1 \mathrm{~mA}$ was applied for $20 \mathrm{~min}$ for anodal and cathodal tDCS resulting in a current density of $0.04 \mathrm{~mA} / \mathrm{cm}^{2}$ (Nitsche et al., 2008). To achieve blinding of subjects regarding the type of stimulation, sham stimulation was performed likewise, but the stimulator was automatically turned off after $30 \mathrm{~s}$. As subjects may experience a slight itching sensation at the beginning of tDCS, this procedure ensured that (1) subjects could not discriminate between $\mathrm{tDCS}$ conditions and (2) any itching sensation was similarly present in all three tDCS conditions (Gandiga et al., 2006). Furthermore, the current faded in and out over $8 \mathrm{~s}$ at the start and end of tDCS to keep the itching sensation at the beginning of stimulation to a minimum. During tDCS, which was applied in a quiet, dimly lit room, subjects kept their eyes closed.

MRI parameters. For structural images at high resolution, a T1weighted magnetization-prepared, rapid gradient echo pulse sequence was used with the following parameters: FoV read $256 \mathrm{~mm}$, FoV phase $100 \%$, slice thickness $1.00 \mathrm{~mm}, \mathrm{TR}=2250 \mathrm{~ms}, \mathrm{TE}=3.03 \mathrm{~ms}$, distance factor $50 \%$, orientation sagittal, flip angle $9^{\circ}, 176$ slices (as in Moos et al., 2012).

Anatomically guided neuronavigation based upon cytoarchitectonical probability maps. Based on the individual structural MR images, frameless stereotactical neuronavigation using the Polaris System (Northern Digital) and the software package Brainsight (Rogue Research) were applied for electrode placement (Sparing et al., 2008) after each subject's individual T1-weighted MRI was coregistered to anatomical surface landmarks of the subject's head.

In a first step, the left IPL was identified by macroanatomical landmarks (e.g., the Silvian fissure, the postcentral sulcus, and the intraparietal sulcus). As the supramarginal and angular gyri of the IPL seem to be involved in gesture processing to a similar extent (Goldenberg and Hagmann, 1997; Hermsdörfer et al., 2001; Goldenberg and Karnath, 2006; Dovern et al., 2011), we targeted the border zone between these two parts of the IPL for stimulation electrode placement in each subject (Fig. 2). However, as the IPL constitutes a mosaic of different functional areas (i.e., the cytoarchitectonically defined areas PF, PFm, and PG) whose borders are not reflected by macroanatomical landmarks (Mars et al., 2011) and show high interindividual variability (Caspers et al., 2006), we used SPM and the Anatomy toolbox (Anatomy toolbox V 1.7 using the map "All Areas_v17_MPM.mat"; Eickhoff et al., 2005) to determine the location of the stimulation site with respect to the cytoarchitectonically defined maximum probability maps (MPMs) of IPL-areas PF, PFm, and PG. To this aim, the coordinates of the stimulation site (based on neuronavigation and macroanatomical landmarks; Fig. 2) were recorded in each subject. Then, the structural MRI of each subject was normalized to the SPM template brain using SPM5 (http://www.fil.ion.ucl.ac. $\mathrm{uk} / \mathrm{spm} / \mathrm{software} / \mathrm{spm} 5$ ) and standard protocols in MATLAB (http://www. mathworks.de/products/matlab). Thereafter, the coordinates of the individual stimulation site (with respect to the structural MRI of each subject) were normalized into MNI space using the transformation matrix derived from the normalization of the individual MRI to the template brain. Based on these normalized MNI coordinates, the location of the individual stimulation site within the MPMs of the cytoarchitectonically defined areas PF, PFm, and PG was determined. In 4 subjects, the individual stimulation site could not be assigned unambiguously to one of the three cytoarchitectonically defined IPL-areas. Thus, these 4 subjects were excluded from further analyses. With respect to the remaining 26 subjects (mean age, $24.6 \pm 3.5$ years, 14 female; Fig. 2), this procedure revealed that the stimulation site of 8 subjects was located in PF (blue dots), whereas the stimulation site of further 8 subjects was located in PFm (red dots). The stimulation site of the remaining 10 subjects was located in PG (yellow dots). The exclusion of 4 subjects interfered slightly with the predefined pseudo-randomization of stimulation orders: the stimulation order "csa" was not applied in the PF group and the stimulation order "sca" was not applied in the PFm group. All the other stimulation orders were similarly present in all three groups.

Data analysis. Statistical analyses were performed using IBM SPSS statistics version 20. Data were analyzed with repeated-measures ANOVAs and are reported at a significance level of $p<0.05$ for all analyses. Where appropriate, degrees of freedom were GreenhouseGeisser corrected. The focus of the analyses was on the modulation of task performance (gesture matching task, person discrimination task) by anodal, cathodal, or sham tDCS as assessed by changes in RTs and error rates after stimulation (compared with RTs and error rates before stimulation). The two variables of interest [i.e., changes (pre-post) of RTs and error rates] were subjected to a three-factorial ANOVA with the withinsubject factors STIMULATION (three levels: anodal tDCS, cathodal tDCS, and sham tDCS) and TASK (two levels: gesture matching task and person discrimination task) as well as the between-subject factor stimulation SITE (three levels: the IPL-areas PF, PFm, and PG). Trials in which subjects erred were excluded from the RT analysis. The overall error rates were $\sim 3 \%$.

\section{Results}

As the main focus of the current study was on the differential effects of anodal tDCS, cathodal tDCS, and sham tDCS applied over the left IPL on gesture processing (compared with person discrimination as control), the analyses concentrated on the changes of error rates and RTs when task performance before anodal/cathodal/sham tDCS was compared with task performance after anodal/cathodal/sham tDCS in each session. Therefore, positive values indicate improvement of task performance 
by anodal/cathodal/sham tDCS, whereas negative values reflect worsening of task performance by anodal/cathodal/sham tDCS.

There were no significant differences between the error rates and mean RTs across the six sets when applied before tDCS (one-way ANOVA of error rates before tDCS with the between-subject factor SET: $F_{(5,78)}=1.037, p=0.403, \eta_{\mathrm{p}}{ }^{2}=$ 0.067; one-way ANOVA of RTs before tDCS with the between-subject factor SET: $\left.F_{(5,78)}=1.193 p=0.321, \eta_{\mathrm{p}}^{2}=0.077\right)$.

\section{tDCS effects on error rates}

Overall error rates were $\sim 3 \%$. Furthermore, changes in error rates were $<0.6 \%$ for both tasks and the three tDCS conditions. The ANOVA with the factors STIMULATION, TASK, and SITE revealed no significant changes of error rates (neither significant main effects nor significant interactions; all $F$ values $\leq 0.95$, all $p$ values $\geq 0.402$, all effect sizes $\left.\eta_{\mathrm{p}}^{2} \leq 0.076\right)$.

\section{tDCS effects on RTs}

There were no significant differences between the median RTs before the different tDCS conditions [median RT \pm SEM: before anodal tDCS: $932 \pm 31 \mathrm{~ms}$, before sham tDCS: $899 \pm 22 \mathrm{~ms}$, and before cathodal tDCS: $908 \pm 18 \mathrm{~ms} ; F_{(2,50)}=0.685$, $\left.p=0.500, \eta_{\mathrm{p}}^{2}=0.027\right]$. However, there was a significant difference between the RTs before and after sham stimulation (mean difference pre-post sham tDCS \pm SEM: $46.9 \pm 9.4 \mathrm{~ms}, \mathrm{~T}=4.9, p=0.001$ ) indicative of an unspecific within-session learning effect for sham tDCS.

The interactions TASK by STIMULA$\operatorname{TION}\left[F_{(2,46)}=0.86, p=0.43, \eta_{\mathrm{p}}^{2}=0.04\right]$ and STIMULATION by SITE $\left[F_{(4,46)}=\right.$ $\left.0.94, p=0.45, \eta_{\mathrm{p}}^{2}=0.08\right]$ were not significant. However, the three-way interaction of TASK by STIMULATION by SITE was significant $\left[F_{(4,46)}=3.7, p=0.01, \eta_{\mathrm{p}}^{2}=\right.$ $0.24]$. This significant three-way interaction was explored by conducting three separate two-factorial ANOVAs for each of the stimulus sites (PF, PFm, and PG) using the within-subject factors TASK (gesture, person) and STIMULATION (anodal tDCS, cathodal tDCS, and sham tDCS). Of these three two-factorial ANOVAs, only the one for PFm revealed a significant two-way interaction of TASK $\times$ STIMULATION $\left[F_{(2,14)}=4.23, p=0.04\right.$, $\left.\eta_{\mathrm{p}}^{2}=0.38\right]$, whereas this interaction was not significant in the other two ANOVAs for PF $\left[F_{(2,14)}=1.06, p=0.35, \eta_{\mathrm{p}}^{2}=0.13\right]$ and PG $\left[F_{(2,18)}=\right.$ $\left.0.758, p=0.45, \eta_{\mathrm{p}}^{2}=0.08\right]$. For the cytoarchitectonically defined IPL-area PFm, the significant two-way interaction was further explored by three planned paired $t$ tests, which revealed a signif-
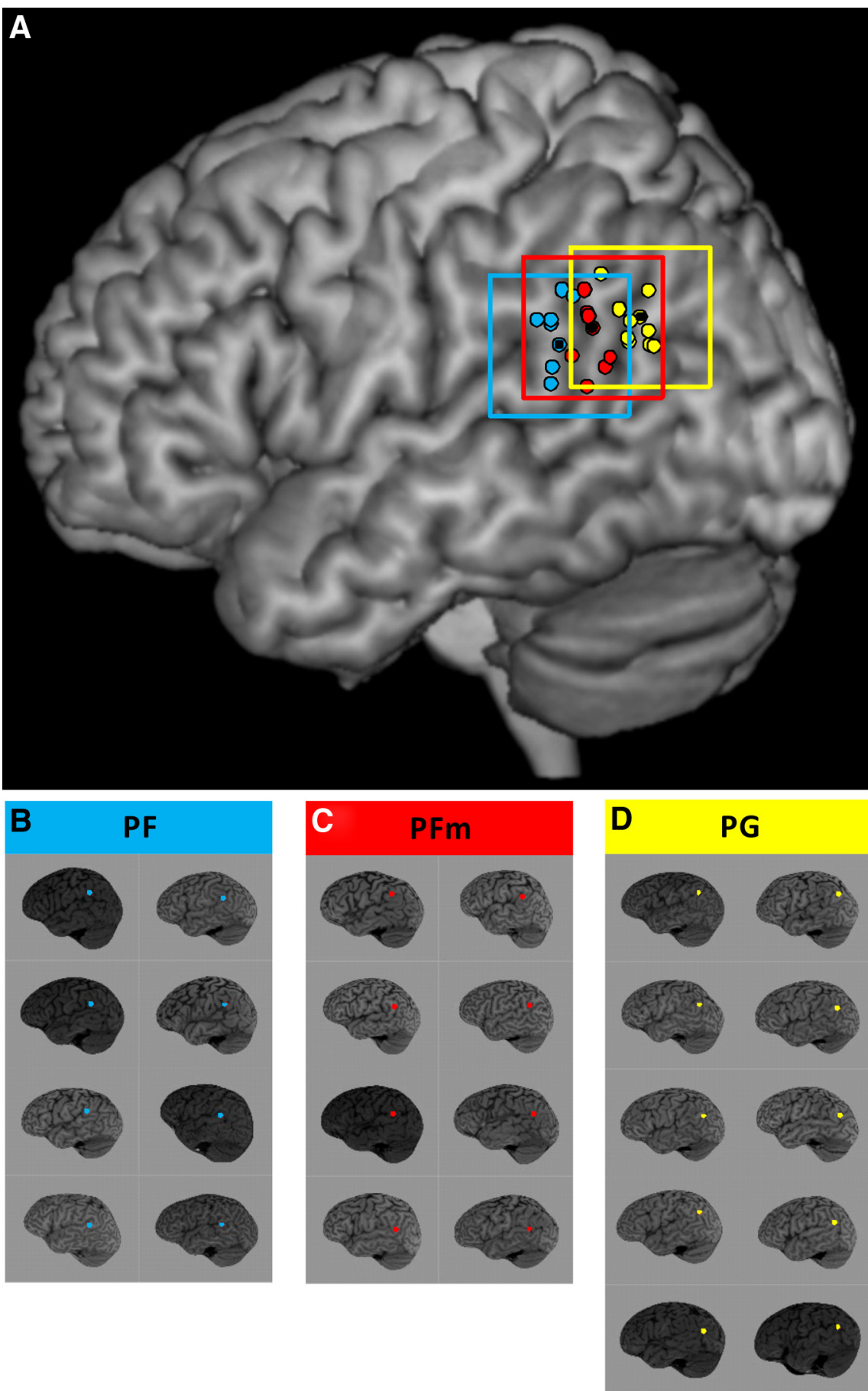

Figure 2. Anatomical location of the individual tDCS sites. All individual stimulation sites for $\operatorname{tDCS}(n=26)$ are displayed on surface renderings of the MNI standard brain $(\boldsymbol{A})$ and the individual subjects' brains $(\boldsymbol{B}-\boldsymbol{D})$. $\boldsymbol{A}$, The individual location of the tDCS electrode's center normalized to MNI standard coordinates and projected onto the surface rendering of the MNI standard template brain are shown for all 26 subjects. The color coding indicates the cytoarchitectonically defined area to which the individual stimulation sites could be assigned using the MPM provided by the Anatomy toolbox in SPM: blue represents PF; red represents PFm; yellow represents PG. In addition, for three selected central stimulation sites within the areas PF, PFm, and PG, a square is depicted that represents the extent of the stimulation electrode used in the currentstudy projected on the surface rendering of the standard brain with the help of the software package Brainsight (Rogue Research). $\boldsymbol{B}-\boldsymbol{D}$, The location of the individual tDCS electrode's center projected onto the surface rendering of each individual, non-normalized brain is depicted for the subjects whose stimulation site was located in the cytoarchitectonically defined area PF ( $\boldsymbol{B}$, blue, left panel, $n=8)$, area PFm (C, red, middle panel, $n=8)$, and area PG (D, yellow, right panel, $n=10)$.

icant difference of the pre-post RTs of the gesture task compared with the pre-post RTs of the person task for anodal tDCS only (mean task difference of pre-post-RTs \pm SEM: $70.8 \pm 18.4 \mathrm{~ms}$, $\mathrm{T}=3.5, p=0.01$, i.e., $p<0.05$, Bonferroni-corrected for the 


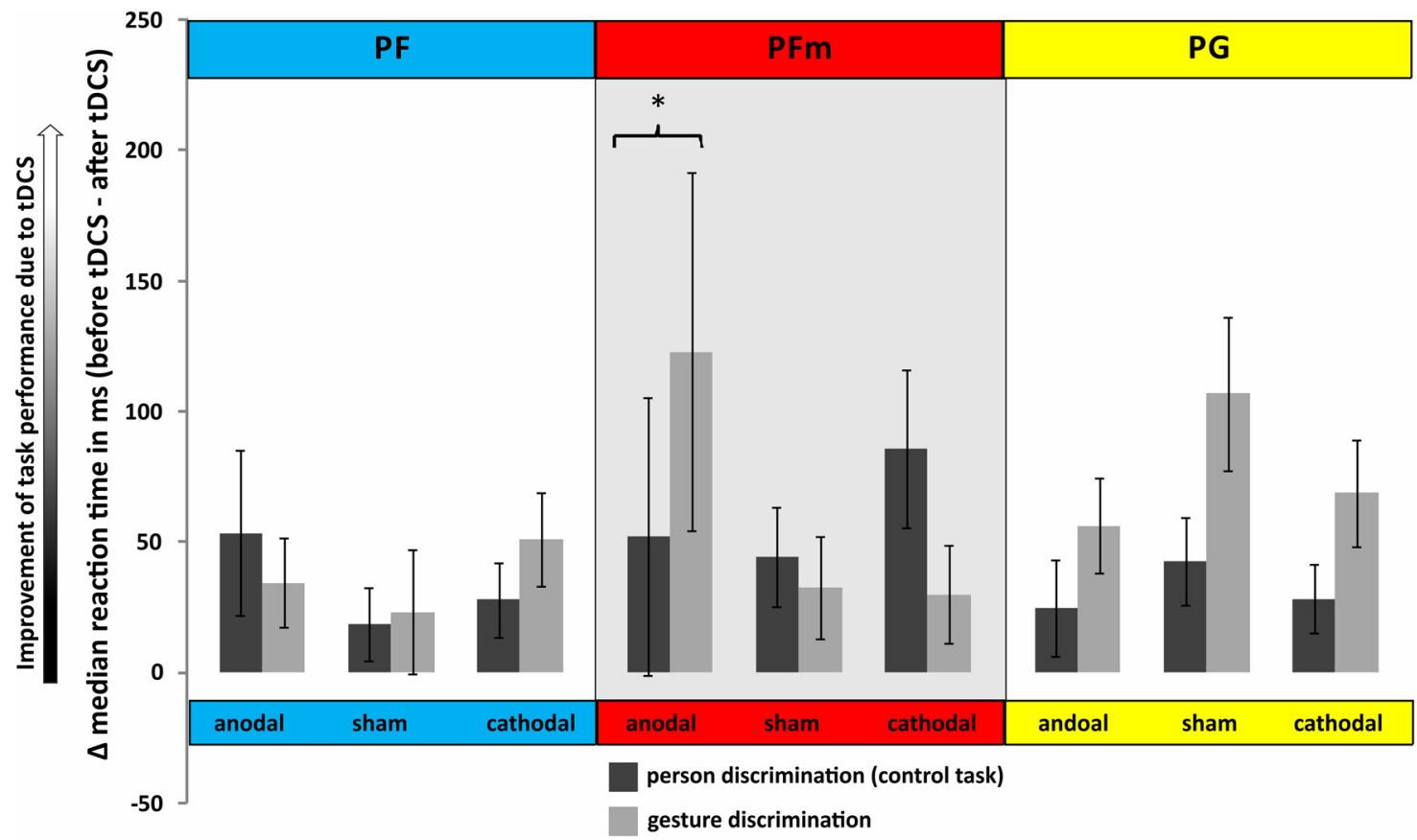

Figure 3. Changes in task performance resulting from tDCS. Changes in task performance induced by tDCS as indexed by changes of RTs for the person discrimination task (dark gray) and the gesture matching task (light gray). Depicted are the changes of RTs when RTs after tDCS are compared with RTs before tDCS for anodal tDCS, sham tDCS, and cathodal tDCS applied over the three cytoarchitectonically defined areas PF (blue, left part), PFm (red, middle part), and PG (yellow, right part) of the left IPL. Positive values indicate an improvement of task performance resulting from anodal $t D C S$, sham $t D C S$, or cathodal tDCS, whereas negative values would indicate a worsening of task performance resulting from tDCS. Further exploration of the significant three-way interaction between TASK, STIMULATION, and SITE by three two-way ANOVAs for each of the three stimulus sites separately (PF, PFm, and PG), and planned paired $t$ tests revealed a differential improvement of the performance in the gesture matching task (compared with the person discrimination task) for anodal tDCS only, but not for sham tDCS or cathodal tDCS (highlighted by the asterisk). Error bars indicate the SEM.

number of tests $(n=3)$; marked with an asterisk in Fig. 3, middle), but not for cathodal tDCS $(-55.9 \pm 39.5 \mathrm{~ms}, \mathrm{~T}=-1.4, p=$ $0.2)$ or sham tDCS $(-12.0 \pm 16.5 \mathrm{~ms}, \mathrm{~T}=-0.7, p=0.5)$. There was a nonsignificant improvement for the person discrimination task following cathodal tDCS over PFm. In summary, the main finding of the current study is a differential improvement of gesture task performance after anodal tDCS over PFm (compared with cathodal tDCS and sham tDCS) as indicated by the significant three-way interaction TASK by STIMULATION by SITE. Furthermore, to control for any learning effects across the three tDCS sessions, stimulation order was included in the above reported ANOVA as a covariate of no interest. This analysis revealed an identical pattern of results and thus ensured that the reported significant $\mathrm{tDCS}$ effects were not due to differences in stimulation order between subjects.

\section{Discussion}

The current findings confirm but also extend previous work suggesting a pivotal role of the left IPL in gesture processing because our data imply that matching of gestures can be facilitated by anodal tDCS when centered over the left IPL-area PFm. As the current results constitute the first evidence that neuronavigated neuromodulation by tDCS can improve gesture matching (already in healthy subjects), they open new avenues for the neurorehabilitation of apraxic patients with deficits in gesture processing.

Previous neuropsychological and neuroimaging studies already highlighted the importance of the IPL for processing gestures, especially (meaningless) hand gestures in the context of imitation (Goldenberg and Hagmann, 1997; Haaland et al., 2000; Hermsdörfer et al., 2001; Decety et al., 2002; Mühlau et al., 2005;
Rumiati et al., 2005; Goldenberg and Karnath, 2006; Tessari et al., 2007; Dovern et al., 2011). The current findings confirm and extend these previous studies as noninvasive neuromodulation by anodal tDCS over the left IPL-area PFm selectively improved performance in the gesture processing task (but not in a person discrimination task, which was used as control). In a previous study, continuous $\theta$ burst stimulation of the left IPL did not result in a modulation of gesture processing (Bohlhalter et al., 2011). In that study, stimulation sites were determined based on the international 10-20-EEG system (see also Bardi et al., 2013). In contrast, we applied anatomically guided neuronavigation for the localization of the stimulation site over the left IPL using frameless stereotactical neuronavigation and cytoarchitectonically defined MPMs provided by the Anatomy toolbox in SPM. Importantly, this allowed detection of a localization-specific tDCS effect in that only anodal tDCS centered over the cytoarchitectonically defined IPL-area PFm facilitated gesture processing, whereas tDCS centered over the IPL-areas PF and PG yielded no significant effect. This finding stresses the importance of neuronavigation for tDCS studies, as previously suggested for TMS (Sparing et al., 2008). The need for neuronavigation is likely to be increased when the stimulated brain region (here: the parietal cortex) exhibits a complex organization with many functionally specific subregions (Grefkes and Fink, 2005). The issue is further complicated when this anatomical/functional subspecialization is not reflected in (macro)anatomical landmarks (Mars et al., 2011) and/or shows a large interindividual variability (Caspers et al., 2006).

Taking into account the size of the stimulation electrode (here: $25 \mathrm{~cm}^{2}$ ) and the rather broad distribution of the tDCS 
current under the stimulation electrode (Wagner et al., 2007), it is unlikely that the observed facilitatory effects of anodal tDCS were due to an isolated stimulation of area PFm. Area PFm lies inbetween areas PF and PG (Fig. 2A). Rather, the data suggest that centering anodal tDCS over area PFm allowed facilitating gesture processing. Whether or not this effect is due to the possibility that this stimulation affected both major parts of the left IPL (i.e., the supramarginal gyrus and the angular gyrus) remains to be further investigated in future studies (e.g., using smaller electrodes enabling more focal stimulation and thereby allowing for more specific statements about the contribution of parietal subareas to task performance). The more rostral (i.e., over area PF) and more caudal (i.e., over area PG) tDCS sites most likely resulted in a stimulation with emphasis on the supramarginal gyrus or the angular gyrus (and possibly other brain regions). This suggestion is consistent with neuropsychological and neuroimaging studies implicating both the supramarginal (Hermsdörfer et al., 2001; Decety et al., 2002; Mühlau et al., 2005) and the angular gyrus (Goldenberg and Hagmann, 1997; Rumiati et al., 2005) in human gesture processing (Tessari et al., 2007; Dovern et al., 2011; Mengotti et al., 2012). Therefore, at present, the most parsimonious interpretation of our tDCS data in the light of previous functional imaging studies is that both parts of the left IPL have to be stimulated simultaneously by anodal tDCS to facilitate matching of hand gestures in healthy subjects.

It remains to be discussed how a neuronally rather unspecific modulation of the membrane potential (as evoked by tDCS) can lead to improvements of a specific motor cognitive function. During tDCS, cortical excitability changes locally due to polaritydependent depolarization (anodal tDCS) or hyperpolarization (cathodal tDCS) (Stagg and Nitsche, 2011). The up to $1 \mathrm{~h}$ or even longer-lasting aftereffects of tDCS, which are NMDA receptordependent, are similar to mechanisms of neuroplasticity [e.g., long-term potentiation (LTP) or long-term depression (LTD)], which are thought to underlie cognitive processes, such as learning and memory formation (Stagg and Nitsche, 2011). tDCS is thought to enhance the strengthening of dynamic task-related synaptic connections, and it has been shown that combined tDCS and (motor) task performance improve task-specific functional connectivity patterns (Polanía et al., 2011). Another study that underlines the task specificity of tDCS demonstrated using resting state fMRI that anodal tDCS applied over the primary motor cortex modulates elements of the cortico-striato-thalamocortical functional motor circuit (Polanía et al., 2012). Thus, the neuronally rather unspecific modulation of the membrane potential evoked by tDCS results in specific changes of task-related (functional) connectivity patterns, which in turn may underlie improvements of the specific function under investigation. In other words, although rather unspecific, tDCS applied to an appropriate target area involved in a given task may lead to improvements in that particular task by modulating task-specific connectivity patterns.

It should be noted that motor RTs can be modulated by tDCS via electrodes in the vicinity of $\mathrm{Cz}$ (e.g., Stagg et al., 2011). Theoretically, our reference electrode over $\mathrm{Cz}$ may have become active and thereby may have influenced the motor RTs of our subjects. However, as the motor response was identical across task conditions and our main finding constitutes an interaction, it is improbable that such a general effect on the motor RTs would have led to a differential effect on task performance as observed in the current study. Moreover, subjects responded with their left hand (i.e., with the hand ipsilateral to the left parietal tDCS electrode). Furthermore, in the anodal tDCS condition, the reference elec- trode over $\mathrm{Cz}$ (if at all active) would have delivered cathodal stimulation. However, such a cathodal stimulation usually results in a slowing of RTs (Jacobson et al., 2012) rather than an acceleration of RTs as observed in the current experiment for anodal tDCS applied over PFm. Nevertheless, the most stringent proof that the position of the reference electrode (here: above $\mathrm{Cz}$ as in Sparing et al., 2009) did not significantly influence our findings would be to repeat the study with another reference electrode position (e.g., contralateral supraorbital ridge as in Stagg et al., 2011; Moos et al., 2012).

In the current study, we used anodal tDCS applied over left parietal cortex to, for the first time, facilitate motor cognitive processing (i.e., gesture matching). This finding clearly extends previous studies in which, to the best of our knowledge, neuromodulation could only be used to impair gesture processing (Bohlhalter et al., 2011). Together with a previous study showing that (facilitatory) anodal tDCS to the right parietal cortex improved line bisection performance in patients with right hemisphere lesions and neglect (Sparing et al., 2009), our data suggest that tDCS is a powerful tool to modulate higher cognitive processing.

Importantly, matching of gestures and gesture execution are similarly impaired in patients with apraxia (Goldenberg, 1999), which implies a general deficit in gesture processing rather than task-specific deficits (Macuga and Frey, 2012). Therefore, the facilitation of gesture matching by anodal tDCS applied over the left IPL-area PFm constitutes a promising approach to improve gesture processing in patients with apraxia. In the current tDCS study, we investigated a gesture matching task because task performance can be assessed by measuring RTs. In contrast, RTs are not well suited to evaluate the imitation of (static) gestures, a task better evaluated by error rates. Unfortunately, however, error rates for imitation show ceiling effects in healthy subjects (Goldenberg, 1996; Goldenberg and Strauss, 2002). Likewise, for the healthy participants enrolled in the current study, no differential tDCS effect on error rates was observed for the gesture matching task, whereas for anodal tDCS over PFm RTs for correctly judging the presented gesture pair improved considerably. For patients with apraxia and deficits in gesture processing, it can thus be expected that anodal tDCS applied over the left IPL will not only accelerate (in terms of shorter RTs) but also improve (in terms of reduced error rates) gesture processing. Taking into account the anatomical specificity of our findings (see above), it can be assumed that only a simultaneous stimulation of both the left supramarginal and the left angular gyrus by facilitatory anodal tDCS will result in improved gesture processing of patients with apraxia.

\section{References}

Bardi L, Kanai R, Mapelli D, Walsh V (2013) Direct current stimulation (tDCS) reveals parietal asymmetry in local/global and salience-based selection. Cortex 49:850-860. CrossRef Medline

Bohlhalter S, Vanbellingen T, Bertschi M, Wurtz P, Cazzoli D, Nyffeler T, Hess CW, Müri R (2011) Interference with gesture production by theta burst stimulation over left inferior frontal cortex. Clin Neurophysiol 122: 1197-1202. CrossRef Medline

Cantagallo A, Maini M, Rumiati RI (2012) The cognitive rehabilitation of limb apraxia in patients with stroke. Neuropsychol Rehabil 22:473-488. CrossRef Medline

Caspers S, Geyer S, Schleicher A, Mohlberg H, Amunts K, Zilles K (2006) The human inferior parietal cortex: cytoarchitectonic parcellation and interindividual variability. Neuroimage 33:430-448. CrossRef Medline

Decety J, Chaminade T, Grèzes J, Meltzoff AN (2002) A PET exploration of the neural mechanisms involved in reciprocal imitation. Neuroimage 15: 265-272. CrossRef Medline 
Donkervoort M, Dekker J, van den Ende E, Stehmann-Saris JC, Deelman BG (2000) Prevalence of apraxia among patients with a first left hemisphere stroke in rehabilitation centres and nursing homes. Clin Rehabil 14:130136. CrossRef Medline

Dovern A, Fink GR, Saliger J, Karbe H, Koch I, Weiss PH (2011) Apraxia impairs intentional retrieval of incidentally acquired motor knowledge. J Neurosci 31:8102-8108. CrossRef Medline

Dovern A, Fink GR, Weiss PH (2012) Diagnosis and treatment of upper limb apraxia. J Neurol 259:1269-1283. CrossRef Medline

Eickhoff SB, Stephan KE, Mohlberg H, Grefkes C, Fink GR, Amunts K, Zilles K (2005) A new SPM toolbox for combining probabilistic cytoarchitectonic maps and functional imaging data. Neuroimage 25:1325-1335. CrossRef Medline

Frey SH, Fogassi L, Grafton ST, Picard N, Rothwell JC, Schweighofer N, Corbetta M, Fitzpatrick SM (2011) Neurological principles and rehabilitation of action disorders: Computation, Anatomy, and Physiology (CAP) model. Neurorehabil Neural Repair 25:S6-S20. CrossRef Medline

Gandiga PC, Hummel FC, Cohen LG (2006) Transcranial DC stimulation (tDCS): a tool for double-blind sham-controlled clinical studies in brain stimulation. Clin Neurophysiol 117:845-850. CrossRef Medline

Goldenberg G (1995) Imitating gestures and manipulating a manikin: the representation of the human body in ideomotor apraxia. Neuropsychologia 33:63-72. CrossRef Medline

Goldenberg G (1996) Defective imitation of gestures in patients with damage in the left or right hemispheres. J Neurol Neurosurg Psychiatry 61: 176-180. CrossRef Medline

Goldenberg G (1999) Matching and imitation of hand and finger postures in patients with damage in the left or right hemisphere. Neuropsychologia 37:559-566. CrossRef Medline

Goldenberg G (2008) Apraxia. In: Handbook of clinical neurology: neuropsychology and behavioral neurology (Goldenberg G, Miller BL, eds). New York: Elsevier.

Goldenberg G, Hagmann S (1997) The meaning of meaningless gestures: a study of visuo-imitative apraxia. Neuropsychologia 35:333-341. CrossRef Medline

Goldenberg G, Karnath HO (2006) The neural basis of imitation is body part specific. J Neurosci 26:6282-6287. CrossRef Medline

Goldenberg G, Strauss S (2002) Hemisphere asymmetries for imitation of novel gestures. Neurology 59:893-897. CrossRef Medline

Grefkes C, Fink GR (2005) The functional organization of the intraparietal sulcus in humans and monkeys. J Anat 207:3-17. CrossRef Medline

Haaland KY, Harrington DL, Knight RT (2000) Neural representation of skilled movement. Brain 123:2306-2313. CrossRef Medline

Hanna-Pladdy B, Heilman KM, Foundas AL (2003) Ecological implications of ideomotor apraxia. Neurology 60:487-490. CrossRef Medline

Hermsdörfer J, Goldenberg G, Wachsmuth C, Conrad B, Ceballos-Baumann AO, Bartenstein P, Schwaiger M, Boecker H (2001) Cortical correlates of gesture processing: clues to the cerebral mechanisms underlying apraxia during the imitation of menaingless gestures. Neuroimage 14: 149-161. CrossRef Medline

Jacobson L, Koslowsky M, Lavidor M (2012) tDCS polarity effects in motor and cognitive domains: a meta-analytical review. Exp Brain Res 216:1-10. CrossRef Medline

Macuga KL, Frey SH (2012) Neural representation involved $n$ observed, imagined, and imitated actions are dissociable and hierarchically organized. Neuroimage 59:2798-2807. CrossRef Medline

Mars RB, Jbabdi S, Sallet J, O’Reilly JX, Croxson PL, Olivier E, Noonan MP, Bergmann C, Mitchell AS, Baxter MG, Behrens TE, Johansen-Berg H, Tomassini V, Miller KL, Rushworth MF (2011) Diffusion-weighted imaging tractography-based parcellation of the human parietal cortex and comparison with human and macaque resting-state functional connectivity. J Neurosci 31:4087-4100. CrossRef Medline

Mengotti P, Corradi-Dell'acqua C, Rumiati RI (2012) Imitation compo- nents in the human brain: an fMRI study. Neuroimage 59:1622-1630. CrossRef Medline

Moos K, Vossel S, Weidner R, Sparing R, Fink GR (2012) Modulation of top-down control of visual attention by cathodal tDCS over right IPS. J Neurosci 32:16360-16368. CrossRef Medline

Mühlau M, Hermsdörfer J, Goldenberg G, Wohlschläger AM, Castrop F, Stahl R, Röttinger M, Erhard P, Haslinger B, Ceballos-Baumann AO, Conrad B, Boecker H (2005) Left inferior parietal dominance in gesture imitation: an fMRI study. Neuropsychologia 43:1086-1098. CrossRef Medline

Nitsche MA, Paulus W (2000) Excitability changes induced in the human motor cortex by weak transcranial direct current stimulation. J Physiol 527:633-639. CrossRef Medline

Nitsche MA, Liebetanz D, Lang N, Antal A, Tergau F, Paulus W (2003) Safety criteria for transcranial direct current stimulation (tDCS) in humans. Clin Neurophysiol 114:2220-2222; author reply 2222-2223. CrossRef Medline

Nitsche MA, Cohen LG, Wassermann EM, Priori A, Lang N, Antal A, Paulus W, Hummel F, Boggio PS, Fregni F, Pascual-Leone A (2008) Transcranial direct current stimulation: state of the art 2008. Brain Stimul 1:206223. CrossRef Medline

Polanía R, Nitsche MA, Paulus W (2011) Modulating functional connectivity patterns and topological functional organization of the human brain with transcranial direct current stimulation. Hum Brain Mapp 32:12361249. CrossRef Medline

Polanía R, Paulus W, Nitsche MA (2012) Modulating cortico-striatal and thalamo-cortical functional connectivity with transcranial direct current stimulation. Hum Brain Mapp 33:2499-2508. CrossRef Medline

Pomeroy V, Aglioti SM, Mark VW, McFarland D, Stinear C, Wolf SL, Corbetta M, Fitzpatrick SM (2011) Neurological principles and rehabilitation of action disorders: Rehabilitation interventions. Neurorehabil Neural Repair 25:33S-43S. CrossRef Medline

Rumiati RI, Weiss PH, Tessari A, Assmus A, Zilles K, Herzog H, Fink GR (2005) Common and differential neural mechanisms supporting imitation of meaningful and meaningless actions. J Cogn Neurosci 17:14201431. CrossRef Medline

Sparing R, Mottaghy FM (2008) Noninvasive brain stimulation with transcranial magnetic or direct current stimulation (TMS/tDCS): from insights into human memory to therapy of its dysfunction. Methods 44: 329-337. CrossRef Medline

Sparing R, Buelte D, Meister IG, Paus T, Fink GR (2008) Transcranial magnetic stimulation and the challenge of coil placement: a comparison of conventional and stereotaxic neuronavigational strategies. Hum Brain Mapp 29:82-96. CrossRef Medline

Sparing R, Thimm M, Hesse MD, Küst J, Karbe H, Fink GR (2009) Bidirectional alterations of interhemispheric parietal balance by non-invasive cortical stimulation. Brain 132:3011-3020. CrossRef Medline

Stagg CJ, Nitsche MA (2011) Physiological basis of transcranial direct current stimulation. Neuroscientist 17:37-53. CrossRef Medline

Stagg CJ, Jayaram G, Pastor D, Kincses ZT, Matthews PM, Johansen-Berg H (2011) Polarity and timing-dependent effects of transcranial direct current stimulation in explicit motor learning. Neuropsychologia 49:800804. CrossRef Medline

Tessari A, Canessa N, Ukmar M, Rumiati RI (2007) Neuropsychological evidence for a strategic control of multiple routes in imitation. Brain 130:1111-1126. CrossRef Medline

Vanbellingen T, Bohlhalter S (2011) Apraxia in neurorehabilitation: classification, assessment and treatment. NeuroRehabilitation 28:91-98. CrossRef Medline

Wagner T, Fregni F, Fecteau S, Grodzinsky A, Zahn M, Pascual-Leone A (2007) Transcranial direct current stimulation: a computer-based human model study. Neuroimage 35:1113-1124. CrossRef Medline 